\title{
One-dimensional inverse scattering problems: an asymmetric two-component wave system framework
}

\author{
Andrew E Yagle \\ Department of Electrical Engineering and Computer Science, The University of Michigan, \\ Ann Arbor, MI 48109-2122, USA
}

Received 1 June 1988, in final form 10 January 1989

\begin{abstract}
Many one-dimensional inverse scattering problems can be formulated as a two-component wave system inverse problem, including inverse problems for lossless and absorbing acoustic and dielectric media. The advantage of doing so is that well known signal processing algorithms with good numerical stability properties can be used to reconstruct such media from either reflection or transmission responses to impulsive or harmonic sources. If the system is asymmetric, i.e. has different reflectivity functions in different directions, transmission data as well as reflection data are required. This paper summarises algorithms for a wide variety of one-dimensional inverse problems, derives some new ones, and presents a simple framework that reveals much about these problems.
\end{abstract}

\section{Introduction}

The one-dimensional inverse scattering problem is fundamental to many areas of physics. Such problems arise in transmission line theory, for acoustic and dielectric media, for lossy, dispersive and viscoelastic media, and for potential scattering of wavefunctions. These problems are formulated in many different ways, making comparison between various approaches difficult.

Often it is not obvious how much data are required to solve such problems, or what parameters can be recovered from a given amount of data. For example, impulse reflection data is sufficient for some lossy media problems, while other problems require transmission data, or data from both ends of the medium. Other problems cannot be solved using time-domain data at all, in which case it is not always clear what data is needed.

This paper proposes the two-component wave system as a common framework for all of these problems. Although its utility for lossless inverse scattering is well known, its applicability to problems involving lossy, absorbing media is less familiar. However, the two-component wave system formulation of the inverse problem for a given medium is shown to be useful in determining the data needed to reconstruct it. Furthermore, we show how well known signal processing algorithms, whose numerical stability has been extensively studied, can be used to reconstruct a medium from either its reflection or transmission responses to impulsive or harmonic data. In all cases we desire an exact (in principle) solution, which includes the effects of dispersion, absorption, attenuation and multiple reflections.

For many problems of interest, including lossy dielectric, absorbing acoustic and viscoelastic media, the system is asymmetric, i.e. has different reflectivity functions for 
waves travelling in opposite directions. Such media require reflection and transmission data from both ends. We show here how harmonic (single-frequency) data may be used as an alternative for some problems. Note that in specifying an 'inverse scattering problem', we only describe a scattering medium. The available data is unspecified; the idea is to determine what data are needed to reconstruct the medium, and then reconstruct it from this data using a standard procedure.

New contributions in this paper include several new algorithms for reconstructing inhomogeneous absorbing acoustic media and lossy dielectric media from their reflection and transmission responses. In addition, a common formalism for lossless and lossy one-dimensional inverse scattering problems is presented that illuminates many common features of these problems.

The paper is organised as follows. Section 2 introduces the asymmetric twocomponent wave system, and shows how it may be solved using the asymmetric Schur, Levinson and lattice algorithms. These algorithms are well known in signal processing, are easily implemented, and their numerical stability has been studied. The lattice algorithm uses transmission data rather than reflection data, and thus may be used as a check on the results of the other algorithms.

Section 3 solves the inverse problem of reconstructing a lossy transmission line; this is a good physical illustration of how the algorithms function, and of how the twocomponent wave system formulation is derived. Sections 4 and 5 consider lossless and absorbing acoustic media and viscoelastic media, showing how various types of media can be reconstructed using a variety of data. Section 6 considers lossless, absorbing and dispersive electromagnetic inverse problems. Section 7 concludes with a summary of results and insights.

\section{Two-component wave system inverse problem and solution}

In this section the common framework for all of the inverse problems considered in this paper is specified. The asymmetric Schur, Levinson and lattice algorithms for solving the inverse problem for this system are specified; these algorithms require reflection and transmission data from both ends of the system. In the special case of a symmetric system, the first two algorithms require only reflection response data, while the third algorithm requires only transmission response data.

\subsection{The asymmetric two-component wave system}

As we shall see in the remaining sections, many one-dimensional inverse scattering problems can be formulated in terms of the following asymmetric two-component wave system:

$$
\frac{\mathrm{d}}{\mathrm{d} z}\left[\begin{array}{c}
D(z, k) \\
U(z, k)
\end{array}\right]=\left[\begin{array}{cc}
-\mathrm{i} k & -r(z) \\
-s(z) & \mathrm{i} k
\end{array}\right]\left[\begin{array}{l}
D(z, k) \\
U(z, k)
\end{array}\right]
$$

Here $D(z, k)$ and $U(z, k)$ are downgoing and upgoing waves, respectively, $r(z)$ and $s(z)$ are reflectivity functions, $z$ is depth (increasing downward), and $k$ may be either frequency or wavenumber.

Equation (2.1) describes the scattering medium illustrated in figure 1. This medium varies smoothly for $0<z<L$, and it is homogeneous (i.e. $r(z)=s(z)=0$ ) for $z<0$ and $z>L$. At the interfaces $z=0$ and $z=L$, there are two possible conditions: (1) 


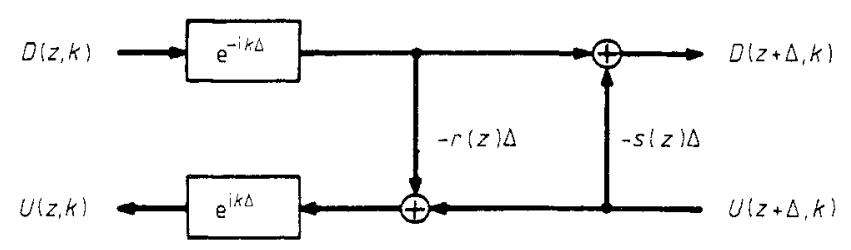

Figure 1. A section of the asymmetric two-component wave system.

$r(z)$ and $s(z)$ are continuous functions; or (2) a free surface (perfect reflector) exists. Both conditions will be used at various points in this paper.

In the time domain (2.1) becomes the pair of equations

$$
\begin{aligned}
& \left(\frac{\partial}{\partial z}+\frac{\partial}{\partial t}\right) \check{D}(z, t)=-r(z) \check{U}(z, t) \\
& \left(\frac{\hat{\partial}}{\partial z}-\frac{\partial}{\partial t}\right) \check{U}(z, t)=-s(z) \check{D}(z, t)
\end{aligned}
$$

where $\check{D}(z, t)=(1 / 2 \pi) \int_{-\infty}^{\infty} D(z, k) \mathrm{e}^{\mathrm{i} k t} \mathrm{~d} k$ is the inverse Fourier transform of $D(z, k)$, and similarly for $\check{U}(z, t)$ (this notation will be used throughout the paper).

$D(z, k)$ and $U(z, k)$ are considered to be waves since $(2.2)$ describe quantities that propagate in increasing and decreasing depth $z$ as $t$ increases. The reflectivity functions $r(z)$ and $s(z)$ describe how much of each wave is reflected into the other wave at each z. If $r(z)=s(z)=0$ then the medium described by (2.1) is locally homogeneous, and no scattering occurs. Note also that the wave speed is assumed to be unity; although this assumption can be relaxed, it complicates matters greatly, as we shall see later.

Sections 3-6 of this paper will formulate a wide variety of inverse scattering problems in the form (2.1). The advantages of doing so are as follows: (1) the form (2.1) has a clear physical interpretation that allows good intuition into the problem; (2) by casting all problems into the same form, similarities between different problems can be exposed, allowing results developed for one problem to be applied to another problem; and (3) standard procedures for solving the inverse problem associated with (2.1) are available, as we now show.

\subsection{Two inverse scattering experiments}

Suppose the system $(2.1)$ is initialised with

$$
\begin{array}{lrr}
D(z, k)=\mathrm{e}^{-i k z} & U(z, k)=R_{1}(k) \mathrm{e}^{\mathrm{i} k z} & z \leq 0 \\
D(z, k)=T_{1}(k) \mathrm{e}^{-i k z} & U(z, k)=0 & z>L .
\end{array}
$$

This is the same as initialising (2.2) with

$$
\begin{array}{ll}
\check{D}(z, t)=\delta(t-z) & \breve{U}(z, t)=\check{R}_{1}(t+z) \quad z \leq 0 \\
\check{D}(z, t)=\check{T}_{1}(t-z) & \breve{U}(z, t)=0 \quad z>L .
\end{array}
$$

Equations (2.3) and (2.4) describe an inverse scattering experiment that consists of probing the medium described by (2.1) and (2.2) with an impulsive plane wave $\delta(t-z)$, incident from above and propagating downward, and getting back a reflection response 


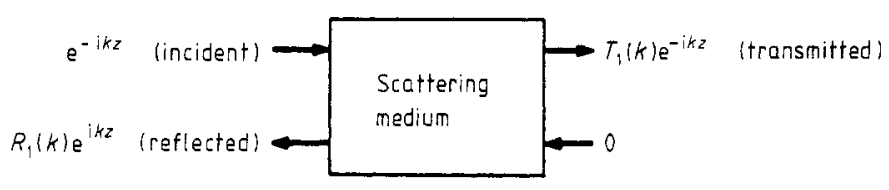

(a)

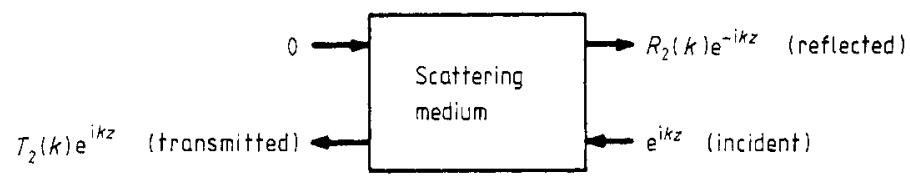

(b)

Figure 2. A scattering experiment with probing from $(a)$ the left and $(b)$ the right.

$\check{R}_{1}(t)$ that is causal and a transmission response $\check{T}_{1}(t)$ at the far end of the medium. This is described in figure $2(a)$.

Since $\check{R}_{1}(t)$ is causal it is clear that $\check{D}(z, t)$ and $\check{U}(z, t)$ have the forms $[1,2]$

$$
\begin{aligned}
& \check{D}(z, t)=\delta(t-z)+\tilde{D}(z, t) 1(t-z) \\
& \check{U}(z, t)=\tilde{U}(z, t) 1(t-z)
\end{aligned}
$$

where $\tilde{D}(z, t)$ and $\tilde{U}(z, t)$ are the smooth parts of $\check{D}(z, t)$ and $\check{U}(z, t)$ (both of which jump at $t=z$ ) and where $1(\cdot)$ is the unit step or Heaviside function. Equations (2.5) are simply a statement of causality.

Now insert equations (2.5) into (2.2), and use a propagation of singularities argument (see [3] for details). Here, this amounts to equating coefficients of $\delta(t-z)$. This yields $[1,2]$

$$
\begin{aligned}
& \left(\frac{\partial}{\partial z}+\frac{\partial}{\partial t}\right) \tilde{D}(z, t)=-r(z) \tilde{U}(z, t) \\
& \left(\frac{\partial}{\partial z}-\frac{\partial}{\partial t}\right) \tilde{U}(z, t)=-s(z) \tilde{D}(z, t) \\
& s(z)=2 \tilde{U}\left(z, z^{+}\right) .
\end{aligned}
$$

The derivation of (2.6) from (2.2) is analogous to the derivation of transport equations for a system of partial differential equations.

If $r(z)=s(z)$, then equations (2.6), initialised using $\tilde{D}(0, t)=0$ and $\tilde{U}(0, t)=\check{R}_{1}(t)$, can be propagated in increasing $z$ and $t$, as noted in [1]. However, if $r(z) \neq s(z)$, then this is not possible. In this case, more data are needed. We now proceed as in [2].

Instead of initialising the system (2.1) at $z=0$ using (2.3), we may, as an alternative, initialise it at $z=L$. In this case the initial conditions are

$$
\begin{array}{lrrl}
U(z, k)=\mathrm{e}^{\mathrm{i} k z} & D(z, k)=R_{2}(k) \mathrm{e}^{-\mathrm{i} k z} & z \geq L \\
U(z, k) & =T_{2}(k) \mathrm{e}^{\mathrm{i} k z} \quad D(z, k)=0 & z<0 .
\end{array}
$$

Equations (2.7) describe an inverse scattering experiment in which the medium is probed with an impulsive plane wave incident from below, and propagating upward. 
This is described in figure $2(b)$. Figures $2(a)$ and $2(b)$ are summarised in the scattering matrix

$$
S=\left[\begin{array}{cc}
T_{1}(k) & R_{1}(k) \\
R_{2}(k) & T_{2}(k)
\end{array}\right]
$$

Now consider the adjoint system to (2.1) [2]

$$
\frac{\mathrm{d}}{\mathrm{d} z}\left[\begin{array}{c}
\bar{D}(z, k) \\
\bar{U}(z, k)
\end{array}\right]=\left[\begin{array}{cc}
-i k & -s(z) \\
-r(z) & i k
\end{array}\right]\left[\begin{array}{l}
\bar{D}(z, k) \\
\bar{U}(z, k)
\end{array}\right] .
$$

The adjoint system is illustrated in figure 3. The term 'adjoint system' first appeared in [4].

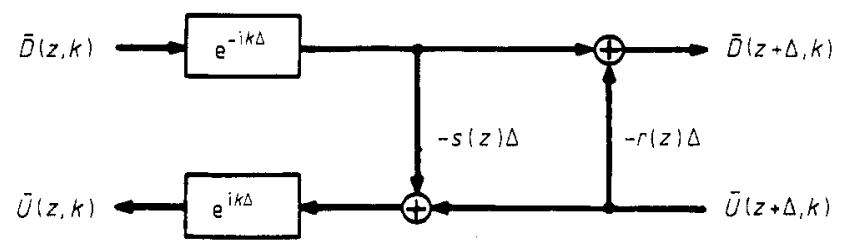

Figure 3. A section of the adjoint asymmetric two-component wave system.

It is not difficult to see that the scattering matrix $S_{\text {adj }}$ for the adjoint system (2.9) is the Hermitian transpose of the inverse of the scattering matrix $S$ [2]:

$$
S_{\mathrm{adj}}=\left(S^{-1}\right)^{\mathrm{H}} \text {. }
$$

This implies a system of equations analogous to (2.6), but with $r(z)$ and $s(z)$ interchanged. These equations are initialised with the $(1,2)$ element of $S_{\text {adj }}$, which is termed $\bar{R}_{1}(k)$.

\subsection{Solution: the asymmetric Schur algorithm}

The significance of introducing the adjoint system (2.9) is that equations (2.6) for the actual system, and the analogous equations for the adjoint system, can be propagated together to reconstruct $r(z)$ and $s(z)$. Discretising depth $z$ and time $t$ into integer multiples of a small constant $\Delta$ and using forward differences, these equations discretise into

$$
\begin{aligned}
& {\left[\begin{array}{l}
D_{1}(z+\Delta, t+\Delta) \\
U_{1}(z+\Delta, t-\Delta)
\end{array}\right]=\left[\begin{array}{cc}
1 & -r(z) \Delta \\
-s(z) \Delta & 1
\end{array}\right]\left[\begin{array}{l}
D_{1}(z, t) \\
U_{1}(z, t)
\end{array}\right] } \\
& {\left[\begin{array}{l}
D_{2}(z+\Delta, t+\Delta) \\
U_{2}(z+\Delta, t-\Delta)
\end{array}\right]=\left[\begin{array}{cc}
1 & -s(z) \Delta \\
-r(z) \Delta & 1
\end{array}\right]\left[\begin{array}{l}
D_{2}(z, t) \\
U_{2}(z, t)
\end{array}\right] } \\
& s(z) \Delta=U_{1}(z, z) / t(z) \\
& r(z) \Delta=U_{2}(z, z) / t(z) \\
D_{1}(N \Delta, N \Delta)= & D_{2}(N \Delta, N \Delta)=t(z=N \Delta)=\prod_{i=0}^{N-1}\left(1-r(i \Delta) s(i \Delta) \Delta^{2}\right) \\
& D_{1}(0, t)=0 \quad U_{1}(0, t)=\check{R}_{1}(t) \\
& D_{2}(0, t)=0 \quad U_{2}(0, t)=\vec{R}_{1}(t) .
\end{aligned}
$$


Equations (2.11) constitute a layer-recursive procedure for reconstructing $r(z)$ and $s(z)$ from the scattering data in the scattering matrix $S$. Note that $\bar{R}_{1}(t)$ can be found from (2.8) and (2.10).

A forward discretisation of (2.6) was used because equations (2.11) first appeared in [5] in the context of fast algorithms for Toeplitz matrix factorisation for signal processing applications. They are called the generalised or asymmetric Schur algorithm. Applied to inverse scattering problems, the continuous version of equations (2.11) first appeared in [2].

There are some subtle differences between the discrete and continuous versions of the Schur algorithm. Note that the factor of 2 in (2.6c) disappears in $(2.11 \mathrm{c})$ and (2.11d). Equations $(2.11 \mathrm{c})$ and $(2.11 d)$ follow from $(2.11 a)$ and $(2.11 b)$ by setting $t=z$ and noting that $U_{i}(z+\Delta, z-\Delta)=0$ by causality. Equation (2.11e) simply propagates $D_{i}(z, z)$ as the transmission loss factor $t(z) ;(2.11 e)$ also follows from $(2.11 a)$ and $(2.11 b)$ by setting $t=z$.

\subsection{Solution: the asymmetric Levinson algorithm}

As an alternative to propagating (2.11), we can use the asymmetric Levinson algorithm. Applied to the inverse scattering problem, this algorithm is [6]

$$
\begin{aligned}
& {\left[\begin{array}{l}
D_{1}(z+\Delta, t+\Delta) \\
U_{1}(z+\Delta, t-\Delta)
\end{array}\right]=\left[\begin{array}{cc}
1 & -r(z) \Delta \\
-s(z) \Delta & 1
\end{array}\right]\left[\begin{array}{l}
D_{1}(z, t) \\
U_{1}(z, t)
\end{array}\right]} \\
& {\left[\begin{array}{l}
D_{2}(z+\Delta, t+\Delta) \\
U_{2}(z+\Delta, t-\Delta)
\end{array}\right]=\left[\begin{array}{cc}
1 & -s(z) \Delta \\
-r(z) \Delta & 1
\end{array}\right]\left[\begin{array}{l}
D_{2}(z, t) \\
U_{2}(z, t)
\end{array}\right]} \\
& s(z=N \Delta) \Delta=\sum_{i=0}^{N-1} D_{1}(z, t=i \Delta) R_{1}(t=(N-i) \Delta) / t(z) \\
& r(z=N \Delta) \Delta=\sum_{i=0}^{N-1} D_{2}(z, t=i \Delta) \bar{R}_{1}(t=(N-i) \Delta) / t(z) \\
& t(z=N \Delta)=\prod_{i=0}^{N-1}\left(1-r(i \Delta) s(i \Delta) \Delta^{2}\right) \\
& D_{1}(0, t)=\delta_{i} \quad U_{1}(0, t)=0 \\
& D_{2}(0, t)=\delta_{t} \quad U_{2}(0, t)=0
\end{aligned}
$$

where $\delta_{t}=1$ if $t=0$ and 0 if $t \neq 0$.

This algorithm also reconstructs $r(z)$ and $s(z)$ from the reflection responses $R_{1}(t)$ and $\bar{R}_{1}(t)$ of the real and adjoint systems. However, the quantities being propagated differ; from the initial conditions it is evident that $D_{i}(z, t)$ and $U_{i}(z, t)$ are the elements of the first column of the state transition matrix of the real and adjoint systems, for $i=1,2$. In fact, the state transition matrix for the real system (2.1) has the form

$$
\left[\begin{array}{ll}
D_{1}(z, k) & U_{2}(z,-k) \\
U_{1}(z, k) & D_{2}(z,-k)
\end{array}\right]
$$

This is discussed in more detail for the symmetric case in [1], and for the asymmetric case in [7]. 
The algorithm (2.12) cannot be parallelised as readily as can (2.11), due to the sum-of-products computations $(2.12 c)$ and $(2.12 d)$. However, the smoothing effects of these two computations may make this algorithm more robust, given noisy data.

\subsection{Asymmetric system with perfect reflectors}

Suppose now that both ends of the system in figure 1 are perfect reflectors. For acoustic media, these are called free or pressure-release surfaces. Note that this is fairly realistic for non-destructive testing, since the impedance contrast between material and air is in the thousands.

The perfect reflectors create a feedback effect, altering the reflection responses from $R_{i}(k)$ to $K_{i}(k)$ and the transmission responses from $T_{i}(k)$ to $X_{i}(k), i=1,2$. The feedback between the reflection response and the perfect reflector implies that $K_{i}(k)=R_{i}(k)+R_{i}^{2}(k)+R_{i}^{3}(k)+\ldots$ and $X_{i}(k)=T_{i}(k)+T_{i}(k) R_{i}(k)+T_{i}(k) R_{i}^{2}(k)+\ldots$, which can be simplified to

$$
\begin{aligned}
& K_{i}(k)=\frac{R_{i}(k)}{1-R_{i}(k)} \quad R_{i}(k)=\frac{K_{i}(k)}{1+K_{i}(k)} \\
& X_{i}(k)=\frac{T_{i}(k)}{1-R_{i}(k)}=T_{i}(k)\left(1+K_{i}(k)\right) \quad T_{i}(k)=\frac{X_{i}(k)}{1+K_{i}(k)} .
\end{aligned}
$$

Identical relations hold for the reflection and transmission responses $\bar{R}_{i}(k), \bar{K}_{i}(k), \bar{T}_{i}(k)$ and $\bar{X}_{i}(k)$ of the adjoint system.

From (2.10) we have $(S)\left(S_{\text {adj }}^{\mathrm{H}}\right)=I$. Writing out the $(i, i)$ element of this for $i=1,2$ gives

$$
T_{i}(k) \bar{T}_{i}(k)^{*}+R_{i}(k) \bar{R}_{i}(k)^{*}=1 \quad i=1,2
$$

and substituting the second of equations (2.13a) and (2.13b) into (2.14) yields

$$
1+K_{i}+\bar{K}_{i}^{*}=X_{i} \bar{X}_{i}^{*} \quad i=1,2 .
$$

Equation (2.15), which is new, states that the reflection response of the system is one side of the cross-correlation of the transmission responses of the system and its adjoint, assuming perfect reflectors at each end.

This result is well known [8] in geophysics for symmetric two-component systems. For symmetric systems, the adjoint system is identical to the original system, and $(2.10)$ becomes a statement that the scattering matrix is unitary. Then (2.14) is a statement of conservation of energy, i.e. symmetric systems are lossless, and (2.15) for symmetric systems is often interpreted as another statement of conservation of energy.

However, it is now clear that this is a misleading interpretation, since asymmetric systems are not lossless (see equation (101) of [2]). Equation (2.15) is new to the literature, although it was mentioned in [7].

\subsection{Solution: the asymmetric lattice algorithm}

The significance of (2.15) is that it allows the lattice algorithm [9] to be applied to the inverse scattering problem for the asymmetric two-component wave system. Unlike the Schur and Levinson algorithms, this algorithm is initialised using transmission responses. 
The algorithm as applied to the inverse scattering problem is [7]

$$
\begin{aligned}
& {\left[\begin{array}{l}
D_{1}(z+\Delta, t+\Delta) \\
U_{1}(z+\Delta, t-\Delta)
\end{array}\right]=\left[\begin{array}{cc}
1 & -r(z) \Delta \\
-s(z) \Delta & 1
\end{array}\right]\left[\begin{array}{l}
D_{1}(z, t) \\
U_{1}(z, t)
\end{array}\right]} \\
& {\left[\begin{array}{l}
D_{2}(z+\Delta, t+\Delta) \\
U_{2}(z+\Delta, t-\Delta)
\end{array}\right]=\left[\begin{array}{cc}
1 & -s(z) \Delta \\
-r(z) \Delta & 1
\end{array}\right]\left[\begin{array}{l}
D_{2}(z, t) \\
U_{2}(z, t)
\end{array}\right]} \\
& s(z=N \Delta) \Delta=\sum_{i=0}^{\infty} D_{1}(z, t=z+i \Delta) \bar{X}_{1}(t=i \Delta) / t(z) \\
& r(z=N \Delta) \Delta=\sum_{i=0}^{\infty} D_{2}(z, t=z+i \Delta) X_{1}(t=i \Delta) / t(z) \\
& t(z=N \Delta)=\prod_{i=0}^{N-1}\left(1-r(i \Delta) s(i \Delta) \Delta^{2}\right) \\
& D_{1}(0, t)=X_{1}(t) \quad U_{1}(0, t)=X_{1}(t) \\
& D_{2}(0, t)=\bar{X}_{1}(t) \quad U_{2}(0, t)=\bar{X}_{1}(t) .
\end{aligned}
$$

Since this algorithm utilises the transmission responses, instead of the reflection responses, of the real and adjoint systems, it may be used as a check on the values of $r(z)$ and $s(z)$ computed using either of the previous algorithms. If the data contain noise, the profiles could be compared to gauge the effects of the noise.

Note that for a symmetric system, corresponding to a lossless medium, the transmission response alone of the medium constitutes sufficient data to reconstruct it [10]. We simply propagate $D_{1}(z, t)=D_{2}(z, t)$ and $U_{1}(z, t)=U_{2}(z, t)$ using $(2.16 a)$, recovering $r(z)=s(z)$ from $(2.16 d)$, and initialised using the transmission response $X(z)$. Note that a perfect reflector is still assumed at the surface $z=0$. Without the perfectly reflecting boundary condition at $z=0$, the transmission response $T(z)$ is still sufficient to reconstruct $r(z)=s(z)$, but very complicated transmutation methods must be used [11].

Note that all three algorithms have recursions that simulate the two-component wave system. However, the quantities being propagated differ, as well as the initialisations and the equations for computing $r(z)$ and $s(z)$. Specifically, the Levinson algorithm propagates the elements of the state transition matrix of the system (2.1), the Schur algorithm propagates the waves associated with the reflection response, and the lattice algorithm propagates the waves associated with the transmission response.

\subsection{Solution: Riccati equations}

Defining the reflection responses of the real and adjoint systems below $z$ as

$$
R_{i}(z, k)=U_{i}(z, k) / D_{i}(z, k) \quad i=1,2
$$

it is easy to show from (2.1) and (2.9) that the $R_{i}(z, k)$ satisfy the Riccati equations [2]

$$
\begin{aligned}
& \frac{\mathrm{d} R_{1}(z, k)}{\mathrm{d} z}=2 i k R_{1}(z, k)+r(z) R_{1}(z, k)^{2}-s(z) \\
& \frac{\mathrm{d} R_{2}(z, k)}{\mathrm{d} z}=2 i k R_{2}(z, k)+s(z) R_{2}(z, k)^{2}-r(z)
\end{aligned}
$$


where $R_{i}(0, k)=R_{i}(k)$. Furthermore, $r(z)$ and $s(z)$ may be computed using

$$
\begin{aligned}
& s(z)=\frac{1}{\pi} \int_{-\infty}^{\infty} R_{1}(z, k) \mathrm{d} k \\
& r(z)=\frac{1}{\pi} \int_{-\infty}^{\infty} R_{2}(z, k) \mathrm{d} k .
\end{aligned}
$$

Equation (2.19a) follows from the forms (2.5) of the waves and the identity (2.6) used to compute $s(z)$ (see equation (3.15) of [1]); similar reasoning leads to $(2.19 b)$. In words, equations (2.19) state that the $t=0$ value of the time-domain reflection response below depth $z$ is due solely to the reflectivity function $r(z)$ or $s(z)$ at depth $z$.

The occurrence of Riccati equations in inverse scattering problems is well known. Note that (2.18) and (2.19) can be propagated together. However, (2.18) should be properly discretised into the layer-removal or dynamic deconvolution equations

$$
\begin{aligned}
& R_{1}(z+\Delta, k)=\mathrm{e}^{2 \mathrm{i} k \Delta} \frac{R_{1}(z, k)-s(z) \Delta}{1-r(z) R_{1}(z, k) \Delta} \\
& R_{2}(z+\Delta, k)=\mathrm{e}^{2 i k \Delta} \frac{R_{2}(z, k)-r(z) \Delta}{1-s(z) R_{2}(z, k) \Delta} .
\end{aligned}
$$

The symmetric $(r(z)=s(z))$ version of $(2.20)$ appears as the layer-removal recursion of [12].

Equations (2.20) can also be derived directly by noting that $R_{1}(z+\Delta, k)$ and $R_{1}(z, k)$ differ by: (1) a time shift, represented by $\mathrm{e}^{2 \mathrm{i} k \Delta}$; (2) the reflection at depth $z$, represented by $s(z) \Delta$; and (3) reverberations between the inhomogeneities at $z$ and $z+\Delta$, represented by the feedback term in the denominator (compare with (2.13)).

It is also possible to associate a coupled pair of Gel'fand-Levitan or Marchenko integral equations to the two-component wave system (2.1) by generalising the argument given in [1] for symmetric systems to asymmetric systems. However, the various fast algorithms given above are much simpler computationally than is the solution of an integral equation.

It is important to emphasise that the discretised algorithms (2.11), (2.12) and (2.16) are the well known Levinson, Schur and lattice algorithms of digital signal processing [4-6]. The fact that the inverse scattering algorithms discretise into well known, numerically stable signal processing algorithms is an important feature of the present work. The Levinson, Schur and lattice algorithms have been shown to be numerically stable (in the sense that they will not diverge for a well-conditioned problem) in [13-15], respectively.

Having reviewed several algorithms for solving the asymmetric two-component wave system (2.1), we spend the rest of this paper formulating a wide variety of onedimensional inverse scattering problems in the form (2.1). Then the material of this section can be used to solve them.

\section{The lossy non-uniform transmission line}

We start off with the lossy non-uniform (LCRG) transmission line, since this problem allows the easiest physical interpretation of the asymmetric two-component wave system, its adjoint and the algorithms of $\S 2$. Much of this material is from [2]; however, 
we also consider a general procedure for formulating problems as asymmetric twocomponent wave systems. Finally, the inverse potential problem for the Schrödinger equation is reformulated as a symmetric two-component wave system.

\subsection{Basic equations for an LCRG transmission line}

An infinitesimal section of length $\Delta$ of an LCRG transmission line is shown in figure 4 where $L(z), C(z), R(z)$ and $G(z)$ are the inductance, capacitance, series resistance and shunt conductance, respectively, per unit length of the line. We assume that wave speed $(L(z) C(z))^{-1 / 2}$ is constant; without loss of generality let $L(z) C(z)=1$.

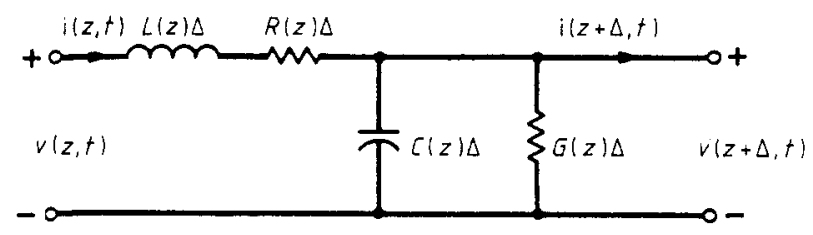

Figure 4. A section of a lossy LCRG non-uniform transmission line.

Kirchoff's voltage and current laws and Fourier transforms result in

$$
\begin{aligned}
& v(z, \omega)=v(z+\Delta, \omega)+(R(z)+\mathrm{i} \omega L(z)) i(z, \omega) \Delta \\
& i(z, \omega)=i(z+\Delta, \omega)+(G(z)+\mathrm{i} \omega C(z)) v(z, \omega) \Delta
\end{aligned}
$$

Divide (3.1) by $\Delta$, let $\Delta \rightarrow 0$, and write the result in matrix form as

$$
\frac{d}{\mathrm{~d} z}\left[\begin{array}{c}
v(z, \omega) \\
i(z, \omega)
\end{array}\right]=\left[\begin{array}{cc}
0 & -(R(z)+\mathrm{i} \omega L(z)) \\
-(G(z)+\mathrm{i} \omega C(z)) & 0
\end{array}\right]\left[\begin{array}{c}
v(z, \omega) \\
i(z, \omega)
\end{array}\right] .
$$

The problem now is to transform system (3.2) into system (2.1). We outline a general procedure for performing this transformation next.

\subsection{Formulation as a two-component wave system}

The first step is to define energy-normalised variables:

$$
\begin{aligned}
& V(z, \omega)=Z^{-1 / 2}(z) v(z, \omega) \\
& I(z, \omega)=Z^{1 / 2}(z) i(z, \omega)
\end{aligned}
$$

using the characteristic impedance $Z(z)=\sqrt{L(z) / C(z)}$. This is generally quite simple for most problems if proper attention is paid to dimensions or energy normalisation.

The next step is to note that for a travelling wave on a lossless transmission line, the voltage $v_{\mathrm{w}}(z, \omega)$ and current $i_{\mathrm{w}}(z, \omega)$ in the frame of reference of the wave are related by

$$
v_{\mathrm{w}}(z, \omega) / i_{\mathrm{w}}(z, \omega)= \pm Z(z) \quad V_{\mathrm{w}}(z, \omega) / I_{\mathrm{w}}(z, \omega)= \pm 1 .
$$

This suggests picking off the variables that are constant in the frame of reference of a travelling wave:

$$
\begin{aligned}
& P(z, \omega)=(V(z, \omega)+I(z, \omega)) / 2 \\
& Q(z, \omega)=(V(z, \omega)-I(z, \omega)) / 2 .
\end{aligned}
$$


Note that $P(z, \omega)=1$ in the frame of reference of a wave travelling in the $+z$ direction, while $Q(z, \omega)=1$ in the frame of reference of a wave travelling in the $-z$ direction. Since this transmission line is lossy, this is not yet sufficient.

Substituting (3.3) and (3.5) in (3.2) gives

$$
\frac{\mathrm{d}}{\mathrm{d} z}\left[\begin{array}{l}
P(z, \omega) \\
Q(z, \omega)
\end{array}\right]=\left[\begin{array}{cc}
-(\mathrm{i} \omega+a(z)) & -m(z)+b(z) \\
-m(z)-b(z) & \mathrm{i} \omega+a(z)
\end{array}\right]\left[\begin{array}{l}
P(z, \omega) \\
Q(z, \omega)
\end{array}\right]
$$

where

$$
\begin{aligned}
& m(z)=\frac{1}{2 Z} \frac{d Z}{\mathrm{~d} z} \\
& a(z)=\frac{1}{2}\left(\frac{R}{L}+\frac{G}{C}\right) \\
& b(z)=\frac{1}{2}\left(\frac{R}{L}-\frac{G}{C}\right) .
\end{aligned}
$$

Now the only problem is the extra $a(z)$ being added to $i \omega$. This can be eliminated by scaling $P(z, \omega)$ and $Q(z, \omega)$ as follows:

$$
\begin{aligned}
& D(z, \omega)=P(z, \omega) \exp \left(\int_{0}^{z} a\left(z^{\prime}\right) \mathrm{d} z^{\prime}\right) \\
& U(z, \omega)=Q(z, \omega) \exp \left(-\int_{0}^{z} a\left(z^{\prime}\right) \mathrm{d} z^{\prime}\right) .
\end{aligned}
$$

Then $D(z, \omega)$ and $U(z, \omega)$ satisfy the asymmetric two-component wave system

$$
\begin{aligned}
& \frac{\mathrm{d}}{\mathrm{d} z}\left[\begin{array}{c}
D(z, k) \\
U(z, k)
\end{array}\right]=\left[\begin{array}{cc}
-\mathrm{i} k & -r(z) \\
-s(z) & \mathrm{i} k
\end{array}\right]\left[\begin{array}{l}
D(z, k) \\
U(z, k)
\end{array}\right] \\
& r(z)=(m(z)-b(z)) \exp \left(2 \int_{0}^{z} a\left(z^{\prime}\right) \mathrm{d} z^{\prime}\right) \\
& s(z)=(m(z)+b(z)) \exp \left(-2 \int_{0}^{z} a\left(z^{\prime}\right) \mathrm{d} z^{\prime}\right)
\end{aligned}
$$

The formulation of the lossy non-uniform transmission line inverse scattering problem as an asymmetric two-component wave system inverse scattering problem is complete. The algorithms of $\S 2$ may be used to reconstruct $r(z)$ and $s(z)$ from the reflection and transmission responses at both ends of the line. The expressions $(3.9 b)$ and $(3.9 c)$ first appeared in [16], in which integral equations, rather than the fast algorithms of $\S 2$, were used to reconstruct them.

\subsection{Interpretation}

Note that if the Heaviside condition for a dispersionless line

$$
R(z) / L(z)=G(z) / C(z)
$$

holds, and constant wave speed $L(z) C(z)=1$ is still assumed, then $b(z)=0$ and each of $L(z), C(z), R(z)$ and $G(z)$ can be reconstructed separately. For such a line, the voltage 
and current propagating in a wave are in phase; no dispersion occurs, and (3.4) now holds exactly, even though the line is lossy. This explains why $(3.9 b)$ and $(3.9 c)$ simplify as much as they do.

For a lossless line $R(z)=G(z)=0$, we have $r(z)=s(z)$, and the two-component wave system is symmetric. This is an important simplification, since reflection data from only one end of the line are now sufficient to reconstruct the line. Alternatively, transmission data alone from the other end of the line are now sufficient to reconstruct it (if the far end of the line is terminated with a perfect reflector, the lattice algorithm may be used; if not, transmutation methods [11] must be used). This is an example of how the type of medium being reconstructed dictates the data required to reconstruct it.

For a transmission line, the concept of waves travelling in opposite directions and being scattered into each other is easy to visualise. The algorithms in $\S 2$ all have clear interpretations in terms of these waves. The adjoint system can be viewed as a fictitious line in which the signs of the inductances and capacitances have been changed. Although the lossy transmission line requires asymmetric scattering, the transformation above has fairly clear physical interpretations at each step. For acoustic media, these physical interpretations are often less obvious, but the same procedure may be used to formulate an asymmetric two-component wave system.

\subsection{Schrödinger equation}

Here the inverse scattering problem for the Schrödinger equation

$$
\left(\frac{\mathrm{d}^{2}}{\mathrm{~d} z^{2}}+k^{2}-V(z)\right) p(z, k)=0
$$

is considered, where the scattering potential $V(z)$ is zero outside the interval $0<z<L$ but is otherwise unknown. Note that $V(z)$ may be either real or complex. Although the potential $V(z)$ is written here solely as a function of $z$, the following procedure also works for a frequency-dependent potential $V(z, k)$; in this case $r(z)$ defined in (3.13) below also becomes frequency dependent, i.e. $r(z, k)$. This will be used in $\$ \S 4.3$ and 5.4 .

Given the boundary conditions (compare with (2.3))

$$
\begin{aligned}
& p(z, k)=\mathrm{e}^{-\mathrm{i} k z}+R(k) \mathrm{e}^{\mathrm{i} k z} \quad z \leq 0 \\
& p(z, k)=T(k) \mathrm{e}^{-i k z} \quad z>L
\end{aligned}
$$

where either the reflection response $R(k)$ or the transmission response $T(k)$ is known, the problem is to compute the scattering potential $V(z)$ from $R(k)$. This problem can be reformulated as a symmetric two-component wave system as follows $[1,2]$.

Let $r(z)$ satisfy the differential equation

$$
r(z)^{2}-\mathrm{d} r / \mathrm{d} z=V(z) \quad 0<z<L
$$

and let $V^{\prime}(z)$ be defined by

$$
V^{\prime}(z)=r(z)^{2}+\mathrm{d} r / \mathrm{d} z \quad 0<z<L .
$$

Now define the associated Schrödinger equation

$$
\left(\frac{\mathrm{d}^{2}}{\mathrm{~d} z^{2}}+k^{2}-V^{\prime}(z)\right) q(z, k)=0
$$


and define its boundary conditions to be

$$
\begin{aligned}
& q(z, k)=\mathrm{e}^{-\mathrm{i} k z}-R(k) \mathrm{e}^{\mathrm{i} k z} \quad z \leq 0 \\
& q(z, k)=T(k) \mathrm{e}^{-i k z} \quad z>L .
\end{aligned}
$$

Then it is not difficult to show that $[1,2]$

$$
\begin{aligned}
& D(z, k)=(p(z, k)+q(z, k)) / 2 \\
& U(z, k)=(p(z, k)-q(z, k)) / 2
\end{aligned}
$$

satisfy a symmetric two-component wave system.

The Schrödinger equation inverse scattering problem may thus be solved as follows. Initialise any of the algorithms of $\S 2$ using the $T(k)$ or $R(k)$ from the Schrödinger boundary condition in (2.3), compute $r(z)$ using these algorithms, and recover $V(z)$ from $r(z)$ using (3.13). The initial condition $r(0)$ is zero if $R(k)$ is strictly proper (i.e. if $\left.\lim _{k \rightarrow \infty} R(k)=0\right)$; if not, there is an immediate reflection at $z=0$. The mapping between $r(z)$ and $V(z)$ specified by (3.13) is not one-to-one; see [17] for more details.

The procedure specified by equations (3.13)-(3.17) can be reversed, to associate a Schrödinger equation with a symmetric two-component wave system (an asymmetric system requires two coupled Schrödinger equations). The Schrödinger equation can then be discretised and propagated as a three-term recurrence, as in [17]. This is the basis of the so-called split algorithms of signal processing, which constitute still another alternative to the algorithms of $\S 2$.

\section{Lossless acoustic media}

In this section algorithms for reconstructing lossless acoustic media from their reflection or transmission responses to impulsive or harmonic point source or plane wave data are derived. Several previous results are presented in a unified framework. Some new algorithms and procedures are derived; these include combining impulsive and harmonic data into a single algorithm, and the use of transmission data for both impulsive and harmonic sources.

\subsection{Lossless acoustic media: basic equations}

The basic equations for lossless acoustic media are

$$
\begin{aligned}
& \nabla \check{p}(x, y, z, t)=-\rho \check{\boldsymbol{a}}(x, y, z, t) \\
& \frac{\partial^{2}}{\partial t^{2}} \check{p}(x, y, z, t)=-\rho c^{2} \nabla \cdot \check{\boldsymbol{a}}(x, y, z, t)
\end{aligned}
$$

where $\check{p}(x, y, z, t)$ is pressure, $\breve{a}(x, y, z, t)$ is medium acceleration, $\rho(x, y, z)$ is density, and $c(x, y, z)$ is wave speed. The acoustic medium is assumed to extend from $z=0$ to $z=L$, and is bounded above and below by infinite homogeneous half-spaces with wave speed $c_{0}$ in the upper half-space.

If $\rho$ and $c$ vary only with depth $z$, and a point source at the origin is used to probe the medium, then the problem has cylindrical symmetry, as illustrated in figure 5. Take Hankel transforms of order zero of $(4.1 b)$ and the $z$ component 
of (4.1a), a Hankel transform of order one of the radial component of $(4.1 a)$, and Fourier transforms of everything (this is sometimes called the Fourier-Bessel transform). Algebraic elimination of the radial component of acceleration results in [18]

$$
\begin{aligned}
& (\partial / \hat{\partial} z) p(z, \xi, \omega)=-\rho(z) a(z, \xi, \omega) \\
& p(z, \xi, \omega) \omega^{2}\left[1-\left(\xi^{2} / \omega^{2}\right) c(z)^{2}\right]=\rho(z) c(z)^{2}(\partial / \partial z) a(z, \xi, \omega)
\end{aligned}
$$

where $p(z, \xi, \omega)$ is the Fourier-Bessel transform of pressure and $a(z, \xi, \omega)$ is the FourierBessel transform of the vertical component of acceleration.

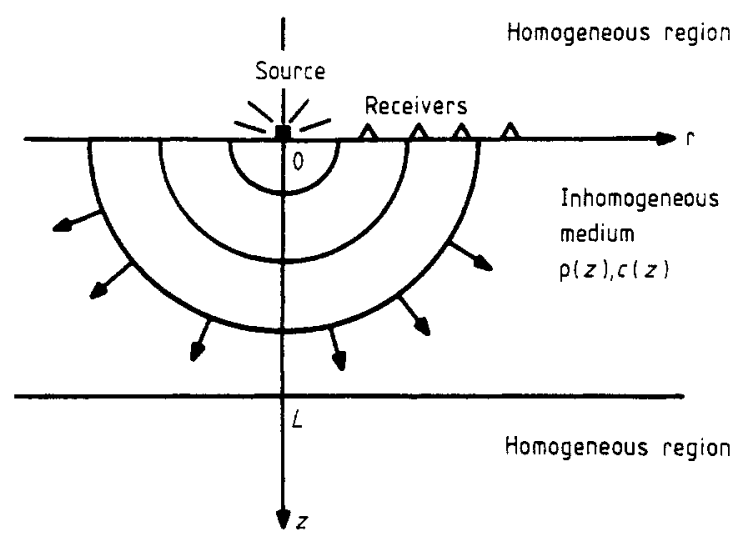

Figure 5. The point source inverse problem for a continuous layered medium.

For a cylindrically symmetric medium, $\xi$ is the radial wavenumber. Define the vertical wavenumber $k_{z}$ by

$$
k_{z}=\left(\frac{\omega^{2}}{c_{0}^{2}}-\xi^{2}\right)^{1 / 2}=\frac{\omega}{c_{0}} \cos \theta
$$

where $c_{0}$ is the wave speed in the homogeneous half-space and $\theta$ is the angle of incidence (measured from the vertical) of a harmonic plane wave. Probing the medium with a point source is equivalent to probing it for all possible angles of incidence $\theta$, including both precritical incidence (real $\theta$ ) and postcritical incidence (imaginary $\theta$ ).

Changing variables from $\xi$ to $k_{z}$ gives

$$
\begin{aligned}
& (\partial / \partial z) p\left(z, k_{z}, \omega\right)=-\rho(z) a\left(z, k_{z}, \omega\right) \\
& p\left(z, k_{z}, \omega\right)\left[k_{z}^{2}+\omega^{2}\left(c(z)^{-2}-c_{0}^{-2}\right)\right]=\rho(z)(\partial / \partial z) a\left(z, k_{z}, \omega\right) .
\end{aligned}
$$

Equations (4.4) are the starting point for our investigations. Note the use of the vertical wavenumber from the start, which is a new approach. Note also that the nature of the point source has not yet been specified.

\subsection{Lossless acoustic media: constant wave speed}

Assume for now that wave speed $c(z)=c_{0}$ is constant. Then (4.4) simplifies to

$$
\begin{aligned}
& (\partial / \partial z) p\left(z, k_{z}, \omega\right)=-\rho(z) a\left(z, k_{z}, \omega\right) \\
& p\left(z, k_{z}, \omega\right) k_{z}^{2}=\rho(z)(\partial / \partial z) a\left(z, k_{z}, \omega\right)
\end{aligned}
$$


It is instructive to compare (4.5) with the lossless version of (3.2) for the transmission line. Proceeding as in $\S 3$, we define energy-normalised pressure and acceleration by dividing and multiplying, respectively, by the square root of density, and then multiplying pressure by $i k_{z}$. Next, we pick off the quantities that are constant on waves (compare with (3.5))

$$
\begin{aligned}
& D\left(z, k_{z}, \omega\right)=\mathrm{i} k_{z} p\left(z, k_{z}, \omega\right) / \sqrt{\rho(z)}+a\left(z, k_{z}, \omega\right) \sqrt{\rho(z)} \\
& U\left(z, k_{z}, \omega\right)=\mathrm{i} k_{z} p\left(z, k_{z}, \omega\right) / \sqrt{\rho(z)}-a\left(z, k_{z}, \omega\right) \sqrt{\rho(z)}
\end{aligned}
$$

and we find that $D\left(z, k_{z}, \omega\right)$ and $U\left(z, k_{z}, \omega\right)$ satisfy the symmetric two-component wave system

$$
\begin{aligned}
& \frac{\mathrm{d}}{\mathrm{d} z}\left[\begin{array}{l}
D\left(z, k_{z}, \omega\right) \\
U\left(z, k_{z}, \omega\right)
\end{array}\right]=\left[\begin{array}{cc}
-\mathrm{i} k_{z} & -r(z) \\
-r(z) & \mathrm{i} k_{z}
\end{array}\right]\left[\begin{array}{c}
D\left(z, k_{z}, \omega\right) \\
U\left(z, k_{z}, \omega\right)
\end{array}\right] \\
& r(z)=\frac{1}{2 \rho(z)} \frac{\mathrm{d} \rho(z)}{\mathrm{d} z}
\end{aligned}
$$

Equations (4.7) imply that the algorithms of $\S 2$ may be used to reconstruct $\rho(z)$ from either the reflection coefficient $R\left(k_{z}\right)$ or the transmission coefficient $T\left(k_{z}\right)$ of the medium. Note that we still have not yet specified what the point source excitation is! However, we now know what is needed: the reflection or transmission response of the medium for all values of vertical wavenumber $k_{z}$. There are several ways of obtaining this; for convenience we refer only to the reflection response, although all statements apply equally well to the transmission response.

(i) Let the point source be an impulse in time, corresponding to an explosive source used to generate acoustic waves. In this case the reflection response $R\left(k_{z}\right)$ is known for all $\omega$, hence for all $k_{z}=\left(\omega / c_{0}\right) \cos \theta$, for a fixed $\theta$. This shows that the impulsive point source problem is overdetermined - the reflection response at only one angle of incidence suffices to reconstruct $\rho(z)$. Note that in the Schur algorithm $z$ is automatically scaled by $c_{0} / \cos \theta$, since this is the effective wave speed through the medium for a plane wave at angle $\theta$. This problem was solved using Schur-like algorithms in $[18,19]$ and elsewhere.

(ii) Let the point source be harmonic in time, corresponding to a narrow-band source used to probe the medium. This approach has been used by Frisk [20] in investigating the ocean bottom. From the Hankel transform of the surface response data, we have $\left\{R\left(k_{z}\right), 0<k_{z}<\omega / c_{0}\right\}$. The lack of high-wavenumber data will result in the loss of high-wavenumber components (i.e. smoothing) in the Fourier transform of the reconstructed $\rho(z)$; this may be acceptable. If not, complex analysis may be used to compute $R\left(k_{z}\right)$ for the missing points on the real axis [21]. This problem was treated in [21] using integral equations, and in [22] using the Schur algorithm.

(iii) In measuring the impulse response of a medium, often the low-frequency components are not excited by the source, and hence not measured. This results in severe errors in the trend of the reconstructed $\rho(z)$ [19]. However, $R\left(k_{z}\right)$ is the pertinent measurement, and it may be obtained in part (low $k_{z}$ ) from point harmonic source data, as in (ii), and in part (high $k_{z}$ ) from impulse response data. Although from different sources, all of these data are used in a single standard inversion procedure (the Schur algorithm). 
The use of transmission data from a harmonic point source is new. The possibility of mixing data from different sources, as in (iii), is also new to the literature. It arises from the recasting of the problem in terms of $k_{z}$, which allows data from a variety of sources to be used in the same inversion procedure. This versatility in using different types of data, reflection or transmission, impulsive or harmonic, is a major justification of the use of the two-component system as a common framework.

\subsection{Lossless acoustic media: varying wave speed}

If the wave speed $c(z)$ is allowed to vary, matters become much more complicated. This is not due to the presence of another parameter to reconstruct (see the results of $\S 5$ below) but is specific to wave speed. The problem is that attempting to transform (4.4) to two-component form, as in $\S 4.2$, leads to reflectivity functions that are functions of $k_{z}$, so that $(2.6 c)$ no longer holds. Each stage of the lattice structure of figure 1 contains transfer functions $r\left(k_{z}, z\right)$ and $s\left(k_{z}, z\right)$ that filter waves travelling in one direction into waves travelling in the opposite direction. Hence a different approach must be used.

For a harmonic point source, the results of $\S 3.4$ may be used. Defining the energy-normalised pressure $\pi=p / \sqrt{\rho}$ and eliminating $a\left(z, k_{z}, \omega\right)$ from (4.4) results in the Schrödinger equation [22]

$$
\left(\frac{\partial^{2}}{\partial z^{2}}+k_{z}^{2}-V(z, \omega)\right) \pi\left(z, k_{z}, \omega\right)=0
$$

where

$$
\begin{aligned}
& \pi\left(z, k_{z}, \omega\right)=-\mathscr{H}_{r \rightarrow \xi}\{p(z, r, \omega) / \sqrt{\rho(z)}\} \\
& V(z, \omega)=\frac{\omega^{2}}{c_{0}^{2}}-\frac{\omega^{2}}{c^{2}(z)}+\frac{1}{Z} \frac{\mathrm{d}^{2} Z}{\mathrm{~d} z^{2}} \\
& Z(z)=1 / \sqrt{\rho(z)}
\end{aligned}
$$

and (4.3) is used in $(4.9 a)$.

The Schrödinger equation (4.8) can then be transformed into a two-component wave system using the procedure specified in $\$ 3.4$. Note that in general there is no closed-form expression for the reflectivity function $r(z, \omega)$ defined in (3.13). The twocomponent wave system is then reconstructed using any of the algorithms of $\S 2$, using either the reflection response or the transmission response, and the potential $(4.9 b)$ is recovered from $r(z, \omega)$ using (3.13).

The use of the reflection response in this way first appeared in [22]; the alternative use of the transmission response is new. Another alternative would be to propagate the Schrödinger equation (4.8) as a three-term recurrence or split algorithm. This approach was used in [23], although the use of the three-term recurrence as a fast algorithm was not then developed in the signal processing literature. The trace method used in [21] used a different discretisation of the Schrödinger equation.

For an impulsive point source, the problem is still overdetermined, as noted above. However, if the plane wave angle of incidence $\theta$ is fixed, then the lateral wavenumber is invariant throughout the medium, and (4.4) simplifies to [18]

$$
\begin{aligned}
& (\hat{\partial} / \partial z) p\left(z, k_{z}, \omega\right)=-\rho(z) a\left(z, k_{z}, \omega\right) \\
& p\left(z, k_{z}, \omega\right) \frac{\omega^{2}}{c(z)^{2}} \cos ^{2} \theta(z)=\rho(z)(\partial / \partial z) a\left(z, k_{z}, \omega\right)
\end{aligned}
$$


where $\theta(z)$ is the local angle of plane wave propagation measured from the vertical. Defining

$$
\begin{aligned}
& c^{\prime}(z)=c(z) / \cos \theta(z)=c(z) /\left[1-\left(c(z)^{2} / c_{0}^{2}\right) \sin ^{2} \theta\right]^{1 / 2} \\
& \tau(z)=\int_{0}^{z} \frac{\mathrm{d} z^{\prime}}{c^{\prime}\left(z^{\prime}\right)} \\
& Z(\tau)=\rho(\tau) c^{\prime}(\tau)
\end{aligned}
$$

and changing variables reduces $(4.10)$ to

$$
\begin{aligned}
& (\partial / \partial \tau) p\left(\tau, k_{z}, \omega\right)=-Z(\tau) a\left(\tau, k_{z}, \omega\right) \\
& p\left(\tau, k_{z}, \omega\right) \omega^{2}=-Z(\tau)(\hat{\partial} / \partial \tau) a\left(\tau, k_{z}, \omega\right) .
\end{aligned}
$$

Note that $k_{z}$ parametrises the dependence on $\theta$.

Equations (4.12) have the same form as equations (4.5), and so they lead to a two-component wave system with the same form as (4.7). However, note that the travel time transformation $(4.11 b)$ amounts to dynamic time warping; this is the time-domain effect of the variable wave speed. Note the complication this introduces, in addition to being an extra quantity to reconstruct.

Once the two-component system has been attained, the algorithms of $\S 2$ may be used to reconstruct $Z(\tau)$ from either reflection or transmission responses. Using plane wave data at two angles of incidence, the profiles $\rho(z)$ and $c(z)$ can be computed [24]. This procedure has been used to reconstruct acoustic media from their reflection responses at two angles of incidence in, for example, [18, 24]; the possible use of transmission data is new. If reflection data are used, the profiles $\rho(z)$ and $c(z)$ can also be computed directly using the Schur algorithm as in [18]; the approach used in [25] is similar. If the Levinson or lattice algorithms are used, $Z(\tau)$ must be computed first.

\section{Lossy acoustic media}

In this section a wide variety of inverse problems for lossy, absorbing acoustic and viscoelastic media are formulated and solved. Several new algorithms are derived. Formulation as a two-component wave system clarifies what data are needed to reconstruct the medium; this is not always obvious from the original equations.

\subsection{Absorbing acoustic media: two-component wave system}

The basic equations for a one-dimensional absorbing acoustic medium are

$$
\begin{aligned}
& \check{p}(z, t)=-\rho(z) c(z)^{2} \frac{\partial \check{u}(z, t)}{\partial z} \\
& \frac{\partial \check{p}(z, t)}{\partial z}=-\rho(z) \frac{\partial^{2} \check{u}(z, t)}{\partial t^{2}}+d(z) \frac{\partial \check{u}(z, t)}{\partial t}
\end{aligned}
$$

where $\breve{p}(z, t)$ is pressure, $\check{u}(z, t)$ is vertical medium displacement and $d(z)$ is the absorption factor. In comparing (5.1) with (4.1), note that medium displacement, rather than acceleration, is being used since medium absorption is proportional to medium velocity (the Maxwell model). 
We assume that wave speed $c(z)$ is constant; without loss of generality let $c(z)=1$. However, density $\rho(z)$ and absorption $d(z)$ may vary with depth. Proceeding as in $\S 4$, we take Fourier transforms, define energy-normalised pressure and displacement by dividing and multiplying, respectively, by the square root of density, and then multiplying displacement by i $\omega$ to get medium velocity. Next, we pick off the quantities that are constant on waves in a lossless medium:

$$
\begin{aligned}
& P(z, \omega)=p(z, \omega) / \sqrt{\rho(z)}+\mathrm{i} \omega u(z, \omega) \sqrt{\rho(z)} \\
& Q(z, \omega)=p(z, \omega) / \sqrt{\rho(z)}-\mathrm{i} \omega u(z, \omega) \sqrt{\rho(z)}
\end{aligned}
$$

and we find that $P(z, \omega)$ and $Q(z, \omega)$ satisfy the system

$$
\begin{aligned}
& \frac{\mathrm{d}}{\mathrm{d} z}\left[\begin{array}{l}
P(z, \omega) \\
Q(z, \omega)
\end{array}\right]=\left[\begin{array}{cc}
-\mathrm{i} \omega-a(z) & -m(z)+a(z) \\
-m(z)-a(z) & \mathrm{i} \omega+a(z)
\end{array}\right]\left[\begin{array}{l}
P(z, \omega) \\
Q(z, \omega)
\end{array}\right] \\
& m(z)=\frac{1}{2 \rho(z)} \frac{\mathrm{d} \rho(z)}{\mathrm{d} z} \\
& a(z)=-d(z) /(2 \rho(z)) .
\end{aligned}
$$

Equations (5.3) have the same form as (3.6) for the lossy transmission line. As in $\S 3$, we define (compare with (3.8))

$$
\begin{aligned}
& D(z, \omega)=P(z, \omega) \exp \left(\int_{0}^{z} a\left(z^{\prime}\right) \mathrm{d} z^{\prime}\right) \\
& U(z, \omega)=Q(z, \omega) \exp \left(-\int_{0}^{z} a\left(z^{\prime}\right) \mathrm{d} z^{\prime}\right) .
\end{aligned}
$$

Then $D(z, \omega)$ and $U(z, \omega)$ satisfy the asymmetric two-component wave system

$$
\begin{aligned}
& \frac{\mathrm{d}}{\mathrm{d} z}\left[\begin{array}{l}
D(z, \omega) \\
U(z, \omega)
\end{array}\right]=\left[\begin{array}{cc}
-\mathrm{i} \omega & -r(z) \\
-s(z) & \mathrm{i} \omega
\end{array}\right]\left[\begin{array}{l}
D(z, \omega) \\
U(z, \omega)
\end{array}\right] \\
& r(z)=(m(z)-a(z)) \exp \left(2 \int_{0}^{z} a\left(z^{\prime}\right) \mathrm{d} z^{\prime}\right) \\
& s(z)=(m(z)+a(z)) \exp \left(-2 \int_{0}^{z} a\left(z^{\prime}\right) \mathrm{d} z^{\prime}\right)
\end{aligned}
$$

Equations (5.5), which are new, are the asymmetric two-component wave system formulation of an inhomogeneous absorbing medium.

\subsection{Absorbing acoustic media: solution}

Now that the inverse scattering problem for a one-dimensional lossy absorbing acoustic medium has been formulated as the inverse scattering problem for the asymmetric twocomponent wave system (5.5), any of the algorithms of $\S 2$ may be used to reconstruct $r(z)$ and $s(z)$ from the reflection and transmission responses at both ends of the medium. From these two reflectivity functions, the profiles $\rho(z)$ and $d(z)$ may be recovered.

This is the first fast algorithm solution of this problem; previous approaches [26$28]$ required the solution of coupled integral equations. Although fast algorithms can 
be derived for these integral equations, it is clearly more perceptive to use algorithms that propagate physical quantities associated with the inverse problem. The possible application of the asymmetric lattice algorithm, initialised using the transmission responses of the actual and adjoint media, is also new.

If the density $\rho(z)$ is also assumed to be constant, an interesting simplification occurs. Although $r(z)$ and $s(z)$ are still unequal, they both are functions of $d(z)$, so that either of $r(z)$ or $s(z)$ may be computed from the other. This implies that reflection data from one end of the line, or transmission data, are sufficient to reconstruct $d(z)$, even though the system (5.5) is asymmetric! The possibility of propagating an asymmetric two-component system when one reflectivity function is a function of the other was noted in [1]; this is the first practical use of this possibility. This is also the first reconstruction of an absorbing acoustic medium from transmission data.

\subsection{Viscoelastic media: basic equations}

The basic equations for a one-dimensional viscoelastic medium are [29]

$$
\begin{aligned}
& \frac{\partial \check{p}(z, t)}{\partial z}=-\rho(z) \frac{\partial^{2} \check{u}(z, t)}{\partial t^{2}} \\
& \check{p}(z, t)=-G_{\mathrm{e}}(z) \frac{\partial \check{u}(z, t)}{\partial z}-\int_{0}^{\infty} \check{G}_{\mathrm{v}}(z, \tau) \frac{\partial^{2} \check{u}(z, t-\tau)}{\partial z \partial t} \mathrm{~d} \tau .
\end{aligned}
$$

Here $G_{\mathrm{e}}(z)$ is the elastic part of the stress-strain modulus and $\check{G}_{v}(z, t)$ is the viscoelastic part of the stress-strain modulus. Note that $\breve{G}_{v}(z, t)$ acts as a memory function in that it relates the present value of stress $-\check{p}(z, t)$ to past values of strain rate $\partial \check{u}(z, t) / \partial t$; it can also be interpreted as a filter or impulse response.

Fourier transforming (5.6) and defining the resolvent $h(z, \omega)$ to $1+i \omega G_{v}(z, \omega) / G_{\mathrm{e}}(z)$ by

$$
\left(G_{\mathrm{e}}(z)+\mathrm{i} \omega G_{v}(z, \omega)\right)(1-h(z, \omega))=G_{\mathrm{e}}(z)
$$

results in

$$
\begin{aligned}
& \frac{\partial p(z, \omega)}{\partial z}=\rho(z) \omega^{2} u(z, \omega) \\
& p(z, \omega)=-\frac{G_{\mathrm{e}}(z)}{1-h(z, \omega)} \frac{\partial u(z, \omega)}{\partial z}=-\frac{\rho(z) c^{2}(z)}{1-h(z, \omega)} \frac{\partial u(z, \omega)}{\partial z}
\end{aligned}
$$

Here

$$
c(z)=\left(G_{\mathrm{e}}(z) / \rho(z)\right)^{1 / 2}
$$

is the local wave speed of propagation through the medium at $z$. Note that acoustic wave speed is dictated solely by the elastic part of the stress-strain modulus; the viscoelastic part produces dispersion and losses.

Equations (5.8) are identical to the lossless acoustic equations (4.1), except for the factor $1-h(z, \omega)$ in $(5.8 b)$. This factor thus characterises the viscoelasticity of the medium since, along with the elastic parameters $\rho(z)$ and $c(z)$, it determines $\breve{G}_{\mathrm{v}}(z, t)$. 


\subsection{Viscoelastic media: harmonic source solution}

We assume that wave speed $c(z)=c_{0}$ is constant. However, the factor $1-h(z, \omega)$ dividing $c(z)^{2}$ in $(5.8 b)$ has the same effect as a wave speed varying in both depth and frequency. This suggests that impulse response data will not be helpful in reconstructing an inhomogeneous viscoelasic medium. Formulation of this problem as a two-component wave system confirms this: the reflectivity functions are functions of frequency as well as of depth.

We must therefore use a harmonic (single-frequency) point source, with the reflection or transmission responses measured over the entire surface of the medium. Since $1-h(z, \omega)$ is similar to a variable wave speed, it should not be surprising that an asymmetric two-component wave system formulation in terms of the vertical wavenumber $k_{z}$ is not possible, as in $\S 4.3$. However, a Schrödinger equation formulation is easy [30] (compare with (4.9))

$$
\left(\frac{\partial^{2}}{\partial z^{2}}+k_{z}^{2}-V(z, \omega)\right) \pi\left(z, k_{z}, \omega\right)=0 .
$$

Here $\pi\left(z, k_{z}, \omega\right)$ and $Z(z)$ are defined in (4.9); the only difference is the potential $V(z, \omega)$, which is now

$$
V(z, \omega)=\frac{\omega^{2}}{c_{0}^{2}} h(z, \omega)+\frac{1}{Z} \frac{\mathrm{d}^{2} Z}{\mathrm{~d} z^{2}} .
$$

The inverse potential problem for $(5.10)$ can be solved as in $\S 3.4$. Note that the density $\rho(z)$ is determined from the low-frequency $\omega \rightarrow 0$ data, while $h(z, \omega)$ is determined as a separate profile for each $\omega$. Thus the harmonic point source data is required over a wide range of frequencies. Note that $h(z, 0)$ cannot be reconstructed at all; this results in a slight bias in the time domain that is easily corrected since the time response is known to decay to zero.

This approach was proposed for reflection data in [30]; the possible reconstruction of an inhomogeneous viscoelastic medium from its transmission response is new, although [31] gave an algorithm for reconstructing a homogeneous viscoelastic medium from its impulsive plane wave transmission response.

Inhomogeneous viscoelastic media with variable wave speed can be treated in the same way. The only difference is that the potential (5.11) is altered to

$$
V(z, \omega)=\frac{\omega^{2}}{c_{0}^{2}}-\frac{\omega^{2}}{c^{2}(z)}(1-h(z, \omega))+\frac{1}{Z} \frac{\mathrm{d}^{2} Z}{\mathrm{~d} z^{2}} .
$$

From (5.12), the wave speed $c(z)$ can evidently be recovered from the reflection data in the limit $\omega \rightarrow \infty$; however, this is a numerically dubious operation.

It is possible to use impulsive plane wave reflection or transmission responses for a special class of viscoelastic media. We must assume that $h(z, \omega)$ and $\rho(z)$ are piecewise constant, corresponding to a discrete-layered medium, and that $\breve{h}(z, t)=\mathscr{F}^{-1}\{h(z, \omega)\}$ decays to zero in less than the two-way travel time through a layer. Given $D(z, \omega)$ and $U(z, \omega)$ at an interface, the reconstruction of $s(z, \omega)$ then amounts to a deconvolution problem, since the next interface will not affect non-zero values of $\breve{h}(z, t)$. This algorithm was derived in [30], extending [29] to multilayered media. 


\subsection{Other comments for acoustic media}

It is important to distinguish (5.5) from the systems which arise from splitting operations on the pressure and displacement. The result of a splitting operation is generally a system similar to (5.3). The problem with (5.3) is as follows. While $P(z, \omega)$ and $Q(z, \omega)$ are wave-like quantities in the sense that they propagate like waves if $m(z)=a(z)=0$, inhomogeneities affect the transmitted waves as well as the reflected waves; there is no interpretation of waves scattered into each other. This makes it much more difficult to reconstruct the medium using (5.3); the signal processing algorithms of $\S 2$ cannot be used.

It should also be noted that diagonalising the matrix associated with (5.1), which was suggested for lossless media in [32], is insufficient. It is necessary to define energynormalised quantities, to take the sums and differences which are constant on plane waves in lossless media, and to scale these using the loss factor as in (5.8).

Finally, there is one type of inhomogeneous lossless medium for which impulsive plane wave response data is useful. A constant- $Q$ lossy acoustic medium is one for which the fraction of energy lost per unit cycle is independent of frequency; this is a fairly realistic model for most solids over seismic frequencies of interest [33-35].

A constant- $Q$ medium can be characterised by a complex but frequency-independent wave speed $c(z)=c_{\mathrm{r}}(z)+\mathrm{i} c_{\mathrm{j}}(z) \operatorname{sgn}(\omega)$. The wave propagator through a layer of thickness $\Delta$ is then

$$
\exp (-\mathrm{i} \omega \Delta / c)=\exp \left(-\mathrm{i} \omega \Delta c_{\mathrm{r}} /|c|^{2}\right) \exp \left(-|\omega| \Delta c_{\mathrm{i}} /|c|^{2}\right)
$$

The first term represents travel time delay through the layer, and the second term represents frequency-dependent attenuation. Furthermore, the complex but frequencyindependent wave speed implies a complex but frequency-independent reflectivity function $r(z)=(1 / c(z))(\mathrm{d} c(z) / \mathrm{d} z)$. The resulting two-component wave system is symmetric and independent of frequency; this allows the medium to be reconstructed from reflection data alone (see [35]).

\section{Electromagnetic inverse problems}

Several lossy and dispersive electromagnetic inverse problems are considered. These problems are in general analogous to those of $\S 5$, allowing the results of $\S 5$ to be applied to these problems as well.

\subsection{Lossy electromagnetic two-component wave system}

Maxwell's equations for a medium with varying electrical permittivity $\varepsilon(z)$ and conductivity $\sigma(z)$, and constant magnetic permeability $\mu_{0}$, are

$$
\begin{aligned}
& \nabla \times \boldsymbol{B}=\mu_{0}\left(\sigma(z) \boldsymbol{E}+\varepsilon(z) \frac{\partial \boldsymbol{E}}{\partial t}\right) \\
& \nabla \times \boldsymbol{E}=-\frac{\partial \boldsymbol{B}}{\partial t}
\end{aligned}
$$


where $\boldsymbol{E}$ and $\boldsymbol{B}$ are electric and magnetic field strengths, respectively. The Fourier transforms of the components of (6.1) transverse to the $z$ axis are

$$
\begin{aligned}
& \frac{\partial B(z, \omega)}{\partial z}=\mu_{0}(\sigma(z)+\mathrm{i} \omega \varepsilon(z)) E(z, \omega) \\
& \frac{\partial E(z, \omega)}{\partial z}=\mathrm{i} \omega B(z, \omega)
\end{aligned}
$$

where $E(z, \omega)$ is the y-component of $E$ and $B(z, \omega)$ is the $x$ component of $\boldsymbol{B}$.

Equations (6.2) describe a plane wave propagating with wave speed $c(z)=$ $\left(\mu_{0} \varepsilon(z)\right)^{-1 / 2}$ and undergoing absorption due to the non-zero conductivity $\sigma(z)$. Changing variables from depth $z$ to travel time $\tau$, where

$$
\tau(z)=\int_{0}^{z}\left(\mu_{0} \varepsilon\left(z^{\prime}\right)\right)^{1 / 2} \mathrm{~d} z^{\prime}
$$

and defining the linear combinations of normalised electric and magnetic field strengths that are constant on lossless plane waves

$$
\begin{aligned}
& P(\tau, \omega)=E(\tau, \omega) / \sqrt{c(\tau)}-B(\tau, \omega) \sqrt{c(\tau)} \\
& Q(\tau, \omega)=E(\tau, \omega) / \sqrt{c(\tau)}+B(\tau, \omega) \sqrt{c(\tau)}
\end{aligned}
$$

we find that $P(\tau, \omega)$ and $Q(\tau, \omega)$ satisfy the system

$$
\begin{aligned}
& \frac{\mathrm{d}}{\mathrm{d} \tau}\left[\begin{array}{l}
P(\tau, \omega) \\
Q(\tau, \omega)
\end{array}\right]=\left[\begin{array}{cc}
-\mathrm{i} \omega-a(\tau) & -m(\tau)-a(\tau) \\
-m(\tau)+a(\tau) & \mathrm{i} \omega+a(\tau)
\end{array}\right]\left[\begin{array}{l}
P(\tau, \omega) \\
Q(\tau, \omega)
\end{array}\right] \\
& m(\tau)=\frac{1}{2 c(\tau)} \frac{\mathrm{d} c(\tau)}{\mathrm{d} \tau} \\
& a(\tau)=\sigma(\tau) /(2 \varepsilon(\tau)) .
\end{aligned}
$$

Equations (6.5) have the same form as (5.3) for absorbing acoustic media. As in $\S 3$, we define (compare with (5.4))

$$
\begin{aligned}
& D(\tau, \omega)=P(\tau, \omega) \exp \left(\int_{0}^{\tau} a\left(\tau^{\prime}\right) \mathrm{d} \tau^{\prime}\right) \\
& U(\tau, \omega)=Q(\tau, \omega) \exp \left(-\int_{0}^{\tau} a\left(\tau^{\prime}\right) \mathrm{d} \tau^{\prime}\right)
\end{aligned}
$$

Then $D(\tau, \omega)$ and $U(\tau, \omega)$ satisfy the asymmetric two-component wave system

$$
\begin{aligned}
& \frac{\mathrm{d}}{\mathrm{d} \tau}\left[\begin{array}{l}
D(\tau, \omega) \\
U(\tau, \omega)
\end{array}\right]=\left[\begin{array}{cc}
-\mathrm{i} \omega & -r(\tau) \\
-s(\tau) & \mathrm{i} \omega
\end{array}\right]\left[\begin{array}{l}
D(\tau, \omega) \\
U(\tau, \omega)
\end{array}\right] \\
& r(\tau)=(m(\tau)+a(\tau)) \exp \left(2 \int_{0}^{\tau} a\left(\tau^{\prime}\right) \mathrm{d} \tau^{\prime}\right) \\
& s(\tau)=(m(\tau)-a(\tau)) \exp \left(-2 \int_{0}^{\tau} a\left(\tau^{\prime}\right) \mathrm{d} \tau^{\prime}\right) .
\end{aligned}
$$


Equations (6.7), which are new, are the asymmetric two-component wave system formulation of electromagnetic wave propagation in an inhomogeneous lossy medium.

\subsection{Lossy electromagnetic solution}

Equations (6.7) have the same form as (5.5) for an absorbing acoustic medium. All of the comments made in $\S 5.2$, including methods of solution using the algorithms of $\S 2$ and novelty of results, apply equally well here. The only difference is that the present problem allows variable wave speed, and hence requires a travel-time transformation.

Although [26-28] briefly noted the analogy between electromagnetic propagation in lossy media and lossy transmission lines, this is the first solution to this problem using either fast algorithms (instead of coupled integral equations) or transmission data for the real medium and its adjoint. The embedding approach used in [36] is closer in spirit to the present procedure, but the clear interpretations of the two-component wave system and the fast algorithms of $\S 2$ are lacking.

If wave speed is constrained to be constant, then (6.7) becomes an asymmetric system with reflectivity functions $r(\tau)$ and $s(\tau)$ that are both functions of $a(\tau)$. Therefore reflection data from one end of the medium, or transmission data, are sufficient to reconstruct $a(\tau)$, even though $(6.7)$ is asymmetric. This is the same simplification that occurred for absorbing acoustic media with constant density.

If conductivity $\sigma(z)=0$, then the medium is lossless. In this case the wave speed profile as a function of travel time $c(\tau)$ can be computed from either reflection data from one end of the medium, or from transmission data. The wave speed as a function of depth $c(z)$ can then be computed from $c(\tau)$.

\subsection{Dispersive media}

In this section the basic equations for a dispersive electromagnetic medium are presented. Neglecting displacement current, two of Maxwell's equations are

$$
\begin{aligned}
& \nabla \times \boldsymbol{E}(\boldsymbol{x}, t)=-\mu(\boldsymbol{x}) \frac{\partial}{\partial t} \boldsymbol{H}(\boldsymbol{x}, t) \\
& \nabla \times \boldsymbol{H}(\boldsymbol{x}, t)=\frac{\partial}{\partial t} \boldsymbol{D}(\boldsymbol{x}, t)
\end{aligned}
$$

where $\boldsymbol{E}(\boldsymbol{x}, t)$ is electric field strength, $\boldsymbol{H}(\boldsymbol{x}, t)$ is magnetic field intensity, $\boldsymbol{D}(\boldsymbol{x}, t)$ is displacement field strength, and $\mu(x)$ is magnetic permeability, which is now allowed to vary with $x \in R^{3}$.

The displacement and electric field strengths are related by [37]

$$
\boldsymbol{D}(\boldsymbol{x}, t)=\varepsilon(\boldsymbol{x}) \boldsymbol{E}(\boldsymbol{x}, t)-\varepsilon(\boldsymbol{x}) \int_{0}^{\infty} \check{h}(\boldsymbol{x}, \tau) \boldsymbol{E}(\boldsymbol{x}, t-\tau) \mathrm{d} \tau
$$

where $\check{h}(\boldsymbol{x}, t)$ is the susceptibility kernel and $\varepsilon(\boldsymbol{x})$ is electrical permittivity. Note that $\check{h}(\boldsymbol{x}, t)$ acts as a memory function in that it relates the present value of displacement to past values of electric field strength; it is entirely analogous to the resolvent for viscoelastic media.

For a medium varying only along the $z$ axis, the Fourier transform of the transverse components of (6.8) are

$$
\begin{aligned}
& \frac{\partial}{\partial z} E(z, \omega)=\mathrm{i} \omega \mu(z) H(z, \omega) \\
& \frac{\partial}{\partial z} H(z, \omega)=\mathrm{i} \omega \varepsilon(z)(1-h(z, \omega)) E(z, \omega)
\end{aligned}
$$


where $E(z, \omega)$ is the $y$ component of $\boldsymbol{E}, H(z, \omega)$ is the $x$ component of $\boldsymbol{H}$, and (6.9) has been used.

Equations (6.10) have exactly the same form as (5.8) for viscoelastic media. Hence the discussion of $\$ 5.4$ applies directly to the inverse electromagnetic problem for dispersive media. This is the first exact procedure for general inhomogeneous dispersive media, although [37] dealt with homogeneous dispersive media and multilayered dispersive media with parametrised susceptibility kernels $\breve{h}(t)$ (e.g. for a Debye medium $\grave{h}(t)=a \mathrm{e}^{-b t}$ for some parameters $\left.a, b\right)$.

\section{Conclusion}

The asymmetric two-component wave system has been used as a framework for a wide variety of one-dimensional inverse scattering problems. The asymmetry allows the treatment of lossy and absorbing media; as expected, such media in general require transmission data as well as reflection data for both ends of the medium. However, the reflectivity functions in the two-component form of the medium specify what types of data are needed. For example, if frequency $\omega$ appears in them, then it is necessary to use a harmonic source so that the frequency dependence becomes parametric. If one can be computed from the other, or if the system is symmetric, then either reflection data or transmission data for either end of the medium may be used to reconstruct it.

It is interesting to note that dispersion and variable wave speed make the inverse problem much harder than with frequency-independent losses. In this case the use of harmonic source data is essential; fortunately the problem can in general be cast as a Schrödinger equation inverse potential problem, which in turn can be recast as a two-component wave system. It is also interesting that so many different problems can be cast in this form, and then solved with the algorithms of $\$ 2$; this allows comparison between results of different problems. No computational results have been presented; these are available in [22], [30] and [35], and this paper is long enough already.

\section{Acknowledgments}

The author would like to thank the referees for their helpful comments. This work was supported in part by the National Science Foundation under grant MIP-8858082.

\section{References}

[1] Bruckstein A M, Levy B C and Kailath T 1985 Differential methods in inverse scattering SIAM J. Appl. Math. 45 312-35

[2] Yagle A E and Levy B C 1985 The Schur algorithm and its applications Acta Appl. Math. 3 255-84

[3] Burridge R 1980 The Gelfand-Levitan, the Marchenko, and the Gopinath-Sondhi integral equations of inverse scattering theory, regarded in the context of inverse impulse-response problems Wave Motion 2 305-23

[4] Carayannis G, Kalouptsidis N and Manolakis D G 1982 Fast recursive algorithms for a class of linear equations IEEE Trans. Acoust., Speech Sig. Proc. ASSP-30 227-39

[5] Rissanen J 1973 Algorithms for triangular decomposition of block Hankel and Toeplitz matrices with application to factoring positive matrix polynomials Math. Comput. 27 147-54

[6] Wiggins R A and Robinson E A 1965 Recursive solution to the multichannel filtering problem $J$. Geophys. Res. $701885-91$

[7] Chan H and Yagle A E 1988 New connections between non-symmetric Toeplitz matrix algorithms and inverse scattering Proc. 1988 Int. Conf. on Acoustics, Speech and Signal Processing (New York, April 1988) (Piscataway, NJ: IEEE) pp 1667-70 
[8] Kunetz G and d'Erceville I 1962 Sur certaines proprieties d'une onde acoustique plane de compression dans un milieu stratifie Annales Geophys. 18 351-9

[9] Cybenko G 1983 A general orthogonalization technique with applications to time series analysis Math. Comput. 40 323-36

[10] Yagle A E 1988 The lattice algorithm of linear prediction applied to the inverse scattering problem given transmission data Proc. 1988 Int. Conf. on Acoustics, Speech and Signal Processing (New York, April 1988) (Piscataway, NJ : IEEE) pp 1655-8

[11] Carroll $\mathrm{R}$ and Santosa $\mathrm{F} 1984$ Impedance profile recovery from transmission data J. Acoust. Soc. Am. $76935-41$

[12] Tolstoy I and Clay C S 1966 Ocean Acoustics (New York: McGraw-Hill)

[13] Cybenko G 1980 The numerical stability of the Levinson-Durbin algorithm for Toeplitz systems of equations SIAM J. Sci. Stat. Comp. 1 303-19

[14] Bultheel A 1979 Toward an error analysis for fast Toeplitz factorization Tech. Report TW 44 Applied Mathematics and Programming Division, Katholieke Universiteit Leuven, Belgium

[15] Cybenko G 1984 The numerical stability of the lattice algorithm for least squares linear prediction problems BIT 24 441-55

[16] Jaulent M 1982 The inverse scattering problem for LCRG transmission lines J. Math. Phys. 23 2286-90

[17] Bruckstein A M and Kailath T 1987 On discrete Schrödinger equations and their two-component wave equation equivalents J. Math. Phys, 28 2914-24

[18] Yagle A E and Levy B C 1984 Application of the Schur algorithm to the inverse problem for a layered acoustic medium $J$. Acoust. Soc. Am. 76 301-8

[19] Carrion P M 1986 On stability of 1D exact inverse methods Inverse Problems 2 1-22

[20] Frisk G V, Doutt J A and Hays E E 1981 Bottom interaction of low-frequency acoustic signals at small grazing angles in the deep ocean J. Acoust. Soc. Am. 69 84-94

[21] Stickler D C 1983 Inverse scattering in a stratified medium J. Acoust. Soc. Am. 74 994-1005

[22] Yagle A E and Levy B C 1985 A fast algorithm solution of the inverse problem for a layered acoustic medium probed with spherical harmonic waves J. Acoust. Soc. Am. 78 729-37

[23] Hooshyar M A and Razavy M 1983 A method for constructing wave velocity and density profiles from the angular dependence of the refiection coefficient J. Acoust. Soc. Am. 73 19-23

[24] Howard M S 1983 Inverse scattering for a layered acoustic medium using the first-order equations of motion Geophysics 48 163-70

[25] Carrion P M 1985 Computation of velocity and density profiles of acoustic media with vertical inhomogeneities using the method of characteristics applied to the slant stacked data J. Acoust. Soc. Am. 77 1370-6

[26] Jaulent M 1976 Inverse scattering problems in absorbing media J. Math. Phys. 17 1351-60

[27] Weston V H 1972 On the inverse problem for a hyperbolic dispersive partial differential equation $J$. Math. Phys. 13 1952-6

[28] Lee C Q 1982 Wave propagation and profile inversion in lossy inhomogeneous media Proc. IEEE 70 219-28

[29] Ammicht E, Corones J P and Krueger R J 1987 Direct and inverse scattering for viscoelastic media $J$. Acoust. Soc. Am. $81827-34$

[30] Yagle A E 1988 Layer stripping approaches to inverse scattering for inhomogeneous viscoelastic media J. Acoust, Soc. Am. 84 1913-23

[31] Karlsson A 1987 Inverse scattering for viscoelastic media using transmission data Inverse Problems 3 691-709

[32] Claerbout J 1976 Fundamentals of Geophysical data Processing (New York: McGraw-Hill)

[33] Rorres C, Bai J, Pedersen P C and Tretiak O J 1985 Inverse acoustic scattering for one-dimensional lossy media J. Acoust. Soc. Am. 78 1851-8

[34] Ganley D C 1981 A method for calculating synthetic seismograms which include the effects of absorption and dispersion Geophys. 46 1100-7

[35] Yagle A E 1987 A layer stripping fast algorithm for reconstructing a lossy layered medium J. Acoust. Soc. Am. 82 927-36

[36] Kristensson G and Krueger R J 1986 Direct and inverse scattering in the time domain for a dissipative wave equation: II. Simultaneous reconstruction of dissipation and phase velocity profiles $J$. Math. Phys. 27 1683-93

[37] Beezley R S and Krueger R J 1985 An electromagnetic inverse problem for dispersive media J. Math. Phys. $26 \quad 317 \ldots 25$ 